\title{
Kritik der Strumaepidemiologie
}

\author{
II. Altersabhängigkeit* \\ R. Gutekunst, C. Schütte, B. Windler und P. C. Scriba \\ Klinik für Innere Medizin (Direktor: Prof. Dr. P. C. Scriba) der Medizinischen Hochschule Lübeck und Kreisgesundheitsamt Ostholstein Eutin
}

Röntgen-Thoraxbilder $(\mathrm{n}=2000)$ des Gesundheitsamtes Eutin wurden ausgewertet, um einen Anhalt für die Strumahäufigkeit in Norddeutschland zu gewinnen. Bis zum 40. Lebensjahr liegt die Häufigkeit bei $5 \%$. Danach steigt die Prävalenz strumaverdächtiger Röntgenbefunde allmählich auf bis zu $25 \%$ und nach dem 65 . Lebensjahr auf über $50 \%$ an. Die angewandte radiologische Methode zeigt offenbar an, daß es auch in Schleswig-Holstein endemische Strumen gibt, was aufgrund des früher gezeigten alimentären Jodmangels auch zu erwarten war.

Insgesamt gilt die Bundesrepublik Deutschland als ein Strumaendemiegebiet mit einer mittleren Kropfhäufigkeit von $15 \%$ bei Rekruten $(5,6,7)$. Dabei bleibt ein unaufgeklärtes, möglicherweise methodisch bedingtes (18) Rätsel, daß nach dem Palpationsbefund (5) 13- bis 15 jährige Schulkinder eine höhere Strumaprävalenz (Knaben 32\%, Mädchen 42\%) zeigen. Bisher wurde ein Nord-Süd-Gefälle mit einer Zunahme der Strumahäufigkeit bei Rekruten von Norden nach Süden auf das Fünfbis Achtfache (6) beschrieben. Aufgrund der täglichen Beobachtungen bei ambulanten und stationären Patienten in unserer Lübecker Umgebung zweifelten wir bald an den niedrigen Zahlen für norddeutsche Erwachsene. Daher wurde in Anlehnung an Schoknecht und Barich (13) versucht, genauere Daten im Hinblick auf die Altersverteilung zu gewinnen.

Dtsch. med. Wschr. 108 (1983), 1016-1018

(c) 1983 Georg Thieme Verlag Stuttgart - New York

\section{Criticism of the epidemiology of goitre.}

\section{Age-dependency}

Chest radiographs $(n=2000)$ of the public health office of Eutin were evaluated for the frequency of goitre in northern West-Germany. Up to the 40th year of age the frequency was $5 \%$. Thereafter the prevalence of goitre-suspect radiographs gradually rose to $25 \%$ and to $50 \%$ after the 65 th year of life. The radiographic method indicates that endemic goitre exists in Schleswig-Holstein as was to be expected due to formerly demonstrated alimentary iodine deficiency.

\section{Methode}

Alle 914 Röntgenbilder des Thorax in postero-anteriorer Projektion, die im Rahmen von Reihenuntersuchungen am Gesundheitsamt Eutin während der Monate November 1978 bis April 1982 von Personen angefertigt wurden, die in Bad Schwartau leben, und alle 1086 Röntgenbilder des gesamten Jahrganges 1976 von Patienten, die in Eutin ansässig sind, wurden ausgewertet. Als Hinweis auf eine Struma wurden folgende Kriterien $(3,12,16)$ gewertet:

1. Einengung, Verdrängung und (oder) Deformation der Trachea,

2. sichere unilaterale oder bilaterale Verbreiterung des oberen Mediastinums,

3. episternaler Weichteilschatten,

4. strumatypische Verkalkungen im oberen Mediastinum.

Nur wenn mindestens zwei Kriterien sicher positiv waren, wurde der Befund "Struma " erhoben. Es zeigte sich, daß fast ausschließlich die ersten beiden Kriterien der Tracheal- und Mediastinalveränderung verwertbar waren.

\footnotetext{
* Mit Unterstützung der Federico Foundation
} 
Abb. 1. Prävalenz strumaverdächtiger röntgenologischer Veränderungen in verschiedenen Altersgruppen.

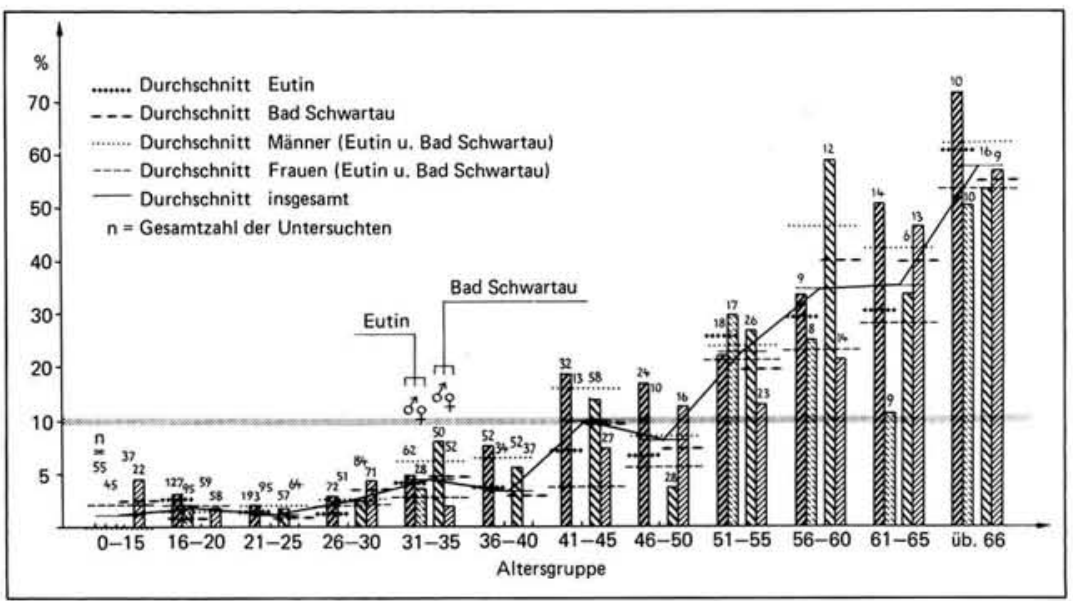

\section{Ergebnisse}

Die Resultate sind komprimiert in der Abbildung 1 wiedergegeben. Wie in anderen Studien die Strumahäufigkeit $(4,5,9,13,17)$, nimmt in der hier vorgelegten Serie die Prävalenz der strumaverdächtigen Veränderungen auf den Schirmbildaufnahmen mit dem Alter zu. Bis zum 40. Lebensjahr bleibt die Häufigkeit unter $5 \%$ und erhöht sich dann auf etwa $10 \%$. Nach dem 50. Lebensjahr steigen die Zahlen sprunghaft auf über $25 \%$ und nach dem 65 . Lebensjahr bis über $50 \%$ an. Das Verhältnis der "Strumahäufigkeit " von Männern und Frauen ist unterschiedlich, jedoch ist insgesamt ein Überwiegen der positiven Röntgenbefunde bei Männern zu beobachten.

Eine Stichprobe $(n=200)$ zeigte, daß vor dem Kriegsausbruch (1939) geborene Einwohner beider Städte nur zu $26 \%$ innerhalb eines Umkreises von $100 \mathrm{~km}$ des jetzigen Wohnortes geboren sind, während bei $32 \%$ der Geburtsort sicher mehr als $100 \mathrm{~km}$ entfernt war. Bei $41,5 \%$ war der Geburtsort nicht mehr feststellbar. - Bei den anderen Altersgruppen liegen Wohn- und Geburtsort nur in wenigen Fällen mehr als $100 \mathrm{~km}$ auseinander. Die Personen mit radiologisch vermuteter Struma waren zu gleichen Teilen weniger $(20 \%)$ oder mehr $(26 \%)$ als $100 \mathrm{~km}$ vom Wohnort entfernt geboren, allerdings konnte der Geburtsort von 54\% der "Strumaträger " aus Datenschutzgründen nicht ermittelt werden.

\section{Diskussion}

Die Auswahl der Probanden ist bei epidemiologischen Untersuchungen kritisch und muß bei jeder Studie aus organisatorischen Gründen letztlich unbefriedigend bleiben. In dieser Untersuchung wurde nur die arbeitende Bevölkerung erfaßt, daher mehr Männer als Frauen. Bei Männern wurde eine höhere "Strumahäufigkeit « festgestellt als bei Frauen, was im Gegensatz zu allen bisherigen Mitteilungen steht $(7,9,15,17)$. Eine sichere Erklärung haben wir nicht. Es darf aber vermutet werden, daß die Kinderzahl der hier erfaßten berufstätigen Frauen deutlich niedriger liegt als in der Durchschnittsbevölkerung. Bekanntermaßen fördert die Schwangerschaft das Strumawachstum (2). Weiter ist möglich, daß berufstätige Frauen früher als nicht berufstätige Frauen medizinisch erfaßt werden, so daß bei ersteren ein Strumawachstum frühzeitiger behandelt würde. Daraus könnte gefolgert werden, daß die wirkliche Strumahäufigkeit in der Gesamtbevölkerung sogar noch höher liegt. Überdies übersieht diese radiologische Methodik kleinere Strumen, die durch Palpation oder Sonographie objektiv nachweisbar werden $(1,7,10,18)$, mit Sicherheit, während umgekehrt mit (10- bis $15 \%$ ) falsch-positiven Röntgenbefunden zu rechnen ist (18). Letztere dürften einen gewissen, zur Zeit nicht exakter quantifizierbaren Beitrag zu den überraschend hohen Werten der "Strumaprävalenz " besonders im Alter beigesteuert haben. Offensichtlich muß die Sensitivität (und Spezifität) der epidemiologischen Methodik für die Untersuchung der Strumahäufigkeit noch überprüft werden, und zwar am besten im direkten Vergleich von Palpation, sonographischer Volumetrie und Schirmbildauswertung (18).

Unsere Resultate erinnern stark an die Untersuchungen von Geiser und Mitarbeitern (4), bei denen eine hohe Strumaprävalenz nach dem 40. Lebensjahr in einem Strumaendemiegebiet mit suboptimaler Jodprophylaxe seit den zwanziger Jahren gefunden wurde. Einerseits hätten wir es also mit der aus Jodprophylaxe-Gebieten bekannten "Verschiebung " der Struma in die späteren Lebensjahrzehnte zu tun, andererseits ist der steile Anstieg der Kropfhäufigkeit in Holstein nach dem 50. Lebensjahr möglicherweise durch zusätzlich zum Jodmangel wirksame Noxen wie kriegsbedingte Fehl- und Mangelernährung zu erklären (7). Vielleicht kommen in dieser Altersgruppe auch noch viele Flüchtlinge aus östlichen Endemiegebieten $(7,8,15)$ mit größerer Strumahäufigkeit zur Geltung. In einer prospektiven Studie müßte gezeigt werden, ob sich die altersbezogene Kurve in den kommenden Jahren abflacht. - Als sicher kann aber gelten, daß es zur Zeit auch in Norddeutschland, und nach der hier vorge- 
legten Studie vermutlich selbst in Schleswig-Holstein, Strumaendemiegebiete gibt. Deshalb bleiben die Bemühungen um eine sichere Strumaprophylaxe so dringlich $(9,11,14)$.

Anmerkung bei der Korrektur: Die für Rostock aufgrund von Schirmbildaufnahmen berichtete niedrigere Strumaprävalenz beruht nach Rücksprache mit einem der Autoren (J. D.) auf einer Selektion (2a).

\section{Literatur}

(1) Brunn, J., U. Block, G. Ruf, I. Bos, W. P. Kunze, P. C. Scriba: Volumetrie der Schilddrüsenlappen mittels Real-time-Sonographie. Dtsch. med. Wschr. 106 (1981), 1338.

(2) Crooks, J., M. I. Tulloch, A. C. Turnbull, D. Davidson, T. Skulasson, G. Snaedal: Comparative incidence of goitre in pregnancy in Iceland and Scotland. Lancet 1967/II, 625 .

(2a) Finger, E., J. Dabels, G. Schünemann, M. Krüger: Strumareihenuntersuchung durch Volksröntgenaktion. Z. Erkrank. Atm.-Org. 158 (1983), 309.

(3) Frey, K. W.: Die Aufgaben der konventionellen Röntgendiagnostik der Schilddrüse. In Scriba, P. C., C. Schneider et al. (Hrsg.): Bildgebende Verfahren in der Schilddrüsendiagnostik. Akt. Endokr. 4, Suppl. 1 (1983), 2
(8) Meng, W., M. Ventz, S. Weber, J. Bednar: Struma und alimentärer Jodmangel in der DDR. Dtsch. Gesundh. Wes. 36 (1981), 1275

(9) Mertz, D. P., K. Tomaras: Rūckgang der Kropfendemie in Südbaden. Münch. med. Wschr. 118 (1976), 497.

(10) Olbricht, T., T. Schmitka, G. Benker, U. Mellinghoff, D. Reinwein: Hāufigkeit von Schilddrüsenkrankheiten in einer Feldstudie. In Scriba, P. C., C. Schneider et al. (Hrsg.): Bildgebende Verfahren in der Schilddrüsendiagnostik. Akt. Endokr. 4, Suppl. 1 (1983), 94.

(11) Pfannenstiel, P., F. A. Horster: Jodmangel in der Bundesrepublik Deutschland. Wirksamkeit (und Risiko) der Kropfprophylaxe durch jodiertes Speisesalz. Dtsch. med. Wschr. 107 (1982), 867.

(12) Schinz, H. R., W. E. Baensch, W. Frommhold, R. Glauner, E. Oehlinger, J. Wellauer (Hrsg.): Lehrbuch der Röntgendiagnostik. 6. Aufl., Bd. IV/2 (Thieme: Stuttgart 1973), $57 \mathrm{ff}$. und 759.

(13) Schoknecht, G., G. Barich: Bestimmung der Häufigkeitsverteilung von Strumen mit Röntgenschirmbildaufnahmen bei Filteruntersuchungen. Dtsch. med. Wschr. 99 (1974), 1860.

(14) Scriba, P. C.: Pathophysiologie der blanden Struma und Jodsalzprophylaxe. Therapiewoche 32 (1982), 29.

(15) Stanbury, J. B., B. S. Hetzel: Endemic Goiter and Endemic Cretinism (J. Wiley \& Sons: New York 1980).

(16) Teschendorf, W., H. Anacker, T. Thurn (Hrsg.): Röntgenologische Differentialdiagnostik. S. Aufl., Bd. 1/2

(Thieme: Stuttgart 1977), 673, 688, 723.

(17) Tunbridge, W. M. G., D. C. Evered, R. Hall, D. Appleton, M. Brewis, F. Clark, J. Grimley-Evans, E. Young, T. Bird, P. A. Smith: The spectrum of thy roid disease in a community. The Wickham survey. Clin. Endocr. 7 (1977), 481.

(18) Waller, D. E., R. Gutekunst, D.

Brossmann, P. C. Scriba: Kritik der Struma-Epidemiologic. I. Strumagröße. Dtsch. med. Wschr. 108 (1983), 1014.

Dr. R. Gutekunst, Dr. C. Schütte, Prof. Dr. P. C. Scriba

Klinik für Innere Medizin

Medizinische Hochschule

2400 Lübeck 1, Ratzeburger Allee 160

Dr. B. Windler

Gesundheitsamt für den Kreis Ostholstein

2420 Eutin, Holstenstr. 52 